Advances in Radio Science (2004) 2: 1-5

(c) Copernicus GmbH 2004

\title{
Determination of quality and storage-time of dielectric mixtures using time domain spectroscopy
}

\author{
O. Schimmer, F. Daschner, M. Kent, and R. Knöchel \\ Microwave Group, Faculty of Engineering, University of Kiel, Germany
}

\begin{abstract}
This paper presents a novel approach for determining certain material properties from permittivity measurements. It does not rely on physical models and mixture formulas. Instead, the reflection time domain response of the material to a step impulse is evaluated in a narrow time window near the steepest ascent of the pulse. A dedicated time domain spectrometer is introduced, which records the data. Principal components are derived directly from the time domain data. The most significant principal components are used to establish a principal component regression formula for prediction of the required material properties. The viability and accuracy of the method is demonstrated by applying it to measurements of the storage time of some chilled fish samples.
\end{abstract}

\section{Introduction}

The market price of many natural materials like for example foodstuffs depends largely on their quality. Although quality is not a very well defined physical feature, it is strongly influenced by properties like composition and storage time. Their determination is thus a very important task in practice. Traditionally, in the food industry quality is specified partly by using food chemical methods and by so called "sensory tests". An example for the latter is the "Quality Index Method" (QIM), which is frequently applied by the fish industry. It employs a panel of skilled individuals, who investigate fish samples with respect to some well defined features. By rating the sample with so called demerit points, and by averaging over the results of a group of persons, a final score is determined, which serves as a quasi-objective tool for grading quality. Clearly, such a method and chemical approaches likewise, are very time consuming and thus costly. Due to the sampling, the results are often not very representative and lack objectivity.

Correspondence to: O. Schimmer

(os@tf.uni-kiel.de)
A solution for the problem could be found by considering the investigated material as a dielectric mixture. It could then be characterised by RF- and microwave-methods for determining dielectric properties in the frequency- or timedomain. Such kinds of approaches are well developed and allow cheap, objective and rapid physical measurements of the permittivity. If only reflection methods are used, single sided access to the material is sufficient offering non-destructive testing, for example by using an open ended coaxial line as the measurement probe. Frequency domain methods measure the reflection coefficient at the end of the coaxial line and evaluate it in order to determine the complex permittivity. This, however, requires high effort of a (dedicated) vector network analyzer. Time domain measurements record the time-domain waveform, but then mostly apply the fourier transform and proceed in the frequency domain, as outlined above. The electronics of traditional time domain reflectometers is also quite sophisticated. However, although the dielectric measurement would provide a solution to the problem of measurement speed, still the difficulty would arise of finding a dependency between changes of the dielectric properties and the storage time of the material or any other quantity which describes the quality.

In the past, attempts have been made, to correlate some of the material properties with the permittivity by using physical models (dielectric mixture formulas). This works for some cases, but has only allowed limited success. The method fails in general, if the complexity of the involved materials increases. The class of substances, into which most natural materials fall, cannot be described by simple models, like for example superpositions of Debye-like relaxation processes. In such materials complicated interactions of the constituents take place, which also depend on history.

This paper presents a measurement set-up and a data processing approach, that offers a unique solution to the described problem: Time domain spectroscopy is applied, but without fourier-transformation of the data. The change of shape of the time domain waveform of a step pulse having a rise time of less than $100 \mathrm{ps}$ is recorded around the steepest 


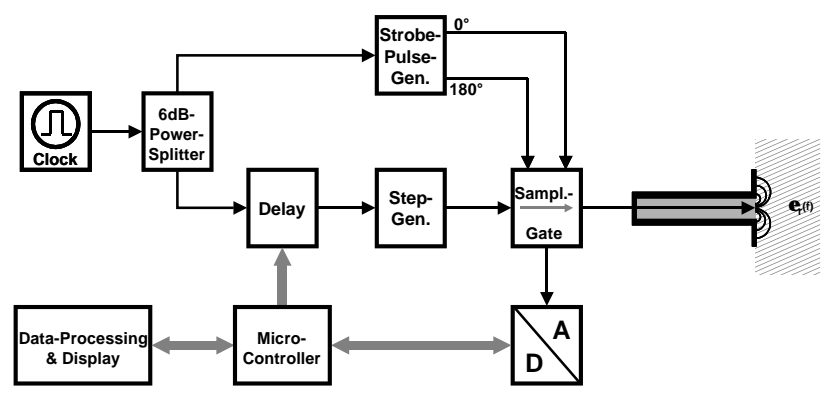

Fig. 1. Block diagram of time domain reflectometer.

ascent in a comparatively small time window of $400 \mathrm{ps,} \mathrm{and}$ then compared to the reflection waveform in air. The sampled time window is too small for application of the fourier transform and determination of the permittivity. That step, in fact, is not required for the present approach! It is based on the insight, that every time domain sampling value already contains information about the dielectric spectrum in a wide frequency range. The sampled part of the pulse is directly processed by applying multivariate statistics, thus allowing direct retrieval of the information contained in the variance of the relaxation spectrum. As will be demonstrated by measurements on fish as an example, this new approach is able to predict changes in storage time or quality (arising from for example, the denaturation of proteins in the material), with high accuracy.

The paper is organised as follows: First the hardware of the developed time domain spectrometer is described. Then the data processing is outlined. Finally, examples of measurements are given, demonstrating the viability of the new approach.

\section{Time domain spectrometer}

The time domain spectrometer acts in a reflectometric mode. It generates and records a step-signal with less than $100 \mathrm{ps}$ rise time and a repetition frequency of $5 \mathrm{MHz}$. Strobe pulses trigger the sampling diodes of a 6-diode travelling wave sampling gate Mulvey (1970), Frye (1971). A holding capacitor is charged during the sampling period and stores the instantaneous voltage for analog-digital conversion. The reflectometer samples the time domain waveform with a time resolution of $10 \mathrm{ps}$ around the steepest ascent of the step function by applying the sequential sampling technique. Various discrete time shifts between the strobe pulses and the measurement signal are achieved by employing a delay line circuit board. This is essential, because the data processing requires the sampling values to be situated at very well defined times. The analog-digital conversion is realised using a commercially available low cost analog-digital-converter. Subsequently, all data is stored in a microcontroller and transferred to a laptop computer for further high level data-processing and for displaying the results.

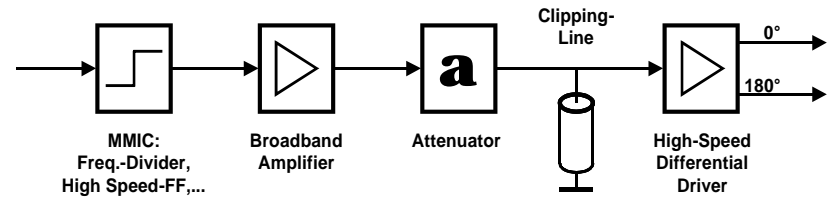

Fig. 2. Strobe pulse generator.

To meet the requirements of the potential users of the instrument, the costs for the time domain spectrometer have to be kept low. To this end and in order to ensure a good reproducibility, the instrument is predominantly built in microstrip-technology and utilises SMD-components. Hence mainly low-cost devices are applied.

\subsection{Hardware overview}

Figure 1 shows the block diagram of the time domain spectrometer. Initially, a crystal oscillator provides the system clock for all the relevant RF circuitry of the system. The clock signal is divided into two channels by a resistive $6 \mathrm{~dB}$ power splitter. The strobe signal is generated in one channel, providing complementary pulses with sufficiently short FWHM-time ${ }^{1}$, in order to trigger the sampling gate. The clock of the second channel can be shifted in time by redirecting the signal over delay lines, which are controlled by the microcontroller. Subsequently, the adjusted clock signal triggers the step generator.

The measurement signal propagates through the sampling gate towards the open ended coaxial line probe and is reflected, due to the material sample at the end. The reflected signal superimposes with the incident step and is acquired in the sampling gate. Finally, the analog signal is converted into digital values by the analog-digital converter, which is triggered and read out by the microcontroller. The controller also buffers the measurements and passes them to a computer for high level data processing and display. The computer permanently communicates with the instrument, in order to control all high level hardware actions.

\subsection{Strobe pulse generator}

The strobe pulse signal is generated with the help of a fast frequency divider, which generates a square-wave signal having a rise-time of approximately $50 \mathrm{ps}$. The pulse is then shaped as shown in Fig. 2. A broadband amplifier increases the pulse amplitude of the signal and isolates the divider from the following circuitry. The amplifier is followed by an attenuator, which suppresses multiple reflections due to inevitable mismatches in the signal channel. Subsequently, the step is transferred into a pulse, using clipping lines Mulvey (1970), Käs and Pauli (1991). A high-speed differential driver outputs complementary strobe pulses with sufficiently high amplitudes, which trigger the sampling gate.

\footnotetext{
${ }^{1}$ full-width half-maximum
} 


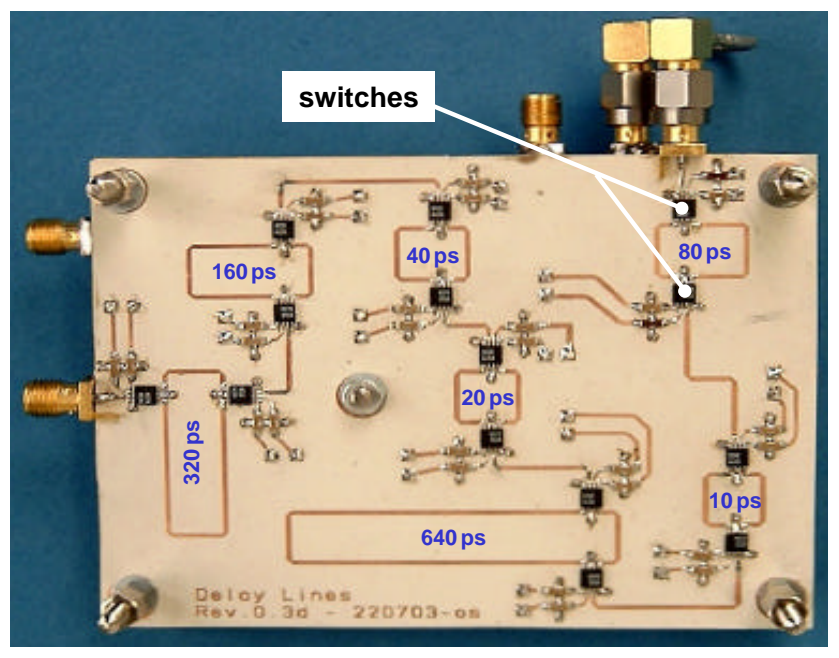

Fig. 3. Delay lines circuit board.

\subsection{Delay lines}

A very simple but also very stable method with respect to absolute time delay but also to jitter performance is achieved by using cascaded delay lines with binary staggered time delays. A photograph of the delay line circuit board is shown in Fig. 3. A "digital" arrangement of $n$ delay lines with binary cascaded lengths provides $N=2^{n}$ equidistant time delays. The present instrument provides a range of delays up to $1.27 \mathrm{~ns}$ with a spacing of $10 \mathrm{ps}$, yielding 128 data points. The switches used are commercially available low-cost RF switches.

\subsection{Step signal generator and sampling gate}

The schematic of the integrated step signal generator and the sampling gate is depicted in Fig. 4. The information about the material under test is obtained from the reflection of the step signal. The latter is easily generated by using a fast switching MMIC frequency divider or high-speed logic components Schimmer and Knöchel (2003). These components have the advantage of a good reproducibility and a good impedance match to the $50 \Omega$-system. In order to adjust the amplitude of the measurement signal, optionally adequate amplifiers or attenuators are applied.

The step signal propagates through the sampling gate towards the probe and is reflected at the aperture plane. The superposition of the incident step signal and the reflection is finally sampled. The utilised travelling wave sampling gate consists of 6 diodes, which become conductive while the leading edges of the complementary strobe pulses simultaneously travel across the two branches of the gate towards the signal line. While the diodes stay conductive, a part of the measurement signal propagates along the diode paths. The trailing edges of the pulses successively close the diodes and a part of the measurement signal is trapped on the microstrip line with length $L_{s}$. That length mainly defines the sampling duration and thus the resolution bandwidth of the

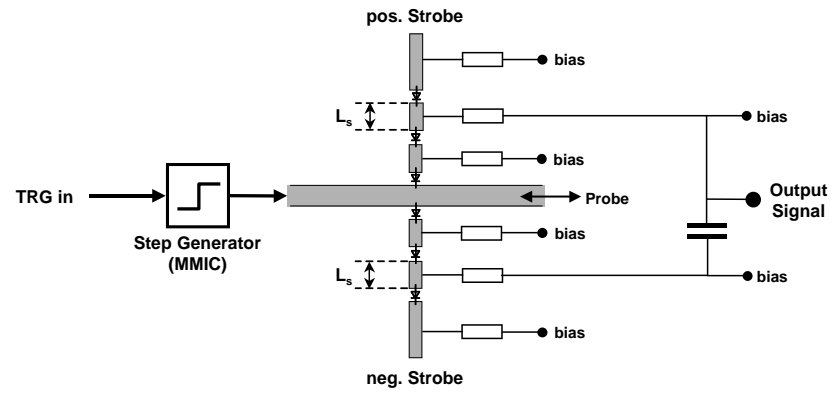

Fig. 4. Step generator and sampling gate.

sampling gate. The trapped portions of the sampled signal subsequently are pre-amplified in an operational amplifier and presented for analog-digital conversion.

\section{Data processing}

Based on the insight, that the information concerning changes of quality is hidden in the variation of the shape of the measured step-signal, one could try to apply a multiple linear regression to the measurement values. However, that approach is difficult to accomplish, because adjacent data points of the steps are highly correlated. That correlation causes a numerical instability of the regression equation Martens and Naes (1989). A decorrelation is achieved and the number of data is reduced by using the structure detecting multivariate statistical approach of principal component analysis (PCA). That method is already widely used in chemometrics Martens and Naes (1989). PCA and principal component regression (PCR) were first introduced in 1996 for the analysis of dielectric data by Kent and Anderson (1996). In order to extract the information about quality and storage time this multivariate approach of principal component analysis (PCA) is applied and both quantities are then expressed in PCR-equations.

Firstly the measured data has to be divided randomly into a calibration and validation group. The principal components (PCs) and the coefficients of the regression equation are determined using the data of the calibration group. The regression equation is then tested using the validation group. For relatively small data sets internal cross validation can be applied Martens and Naes (1989).

The PCs are an orthogonal transformation of the measured data. Hence the collinearity is removed completely. After the transformation a new set of data is available:

$Y_{c}=A \cdot X_{c}$.

$X_{c}$ are the normalised sampled data of the measured stepsignals of the calibration group, $A$ is the transformation matrix and $Y_{c}$ are the new data called principal components. The criterion of the orthogonal transformation is to maximise the variance of the PCs. The PCs are aligned in a way that the first has the greatest variance, the second has the next largest, and so on in in descending order. This criterion is fulfilled 
Table 1. Prediction of the storage time of frozen hake and cod.

\begin{tabular}{lcccc}
\hline Sample & No of PCs & $\mathbf{R}_{\text {adj }}^{2}$ & $\begin{array}{c}\text { PCA } \\
\text { (days) }\end{array}$ & $\begin{array}{c}\text { SEP } \\
\text { (days) }\end{array}$ \\
\hline All whole hake & 6 & 30.8 & 79.1 & 80.7 \\
All Atlantic whole hake & 12 & 73.6 & 55.2 & 58.6 \\
Atlantic winter whole hake & 10 & 99.3 & 10.8 & 11.8 \\
Atlantic summer whole hake & 9 & 93.4 & 12.8 & 14.8 \\
Pacific whole hake & 11 & 95.0 & 16.4 & 19.0 \\
All Atlantic hake fillets & 8 & 66.5 & 43.4 & 45.5 \\
Atlantic winter hake fillets & 8 & 91.2 & 24.6 & 26.3 \\
Atlantic summer hake fillets & 10 & 89.7 & 16.7 & 20.0 \\
All cod & 10 & 89.8 & 32.3 & 34.9 \\
Single frozen cod & 10 & 93.0 & 26.96 & 30.17 \\
\hline
\end{tabular}

when $A$ is composed by the eigenvalues of the covariance matrix of the measured steps.

Normally the first few PCs describe nearly all of the total variance of the data. The last PCs contain only the variance due to the influence of noise. Hence PCA can be used for data reduction purposes. It is also required that the number of PCs used is minimised in order to avoid over fitting. After the transformation some of the first PC's are used as regressors in a multiple linear regression:

$\beta=\left(Y_{c}^{T} Y_{c}\right)^{-1} Y_{c} \cdot \boldsymbol{q}_{c}$

Here, $\beta$ are coefficients of the regression equation and $\boldsymbol{q}_{\boldsymbol{c}}$ are the known quality values of the calibration group. The quality values of the validation group $Q_{v}$ can be estimated after the calibration by the equation

$Q_{v}=\beta \cdot A \cdot X_{v}$

\section{Results}

The prototype instrument described in Sect. 2 was validated with a test series of chill stored cod. The cod was stored on ice up to 18 days. Five samples were available for measurement on each day. The results are shown in Fig. 4. The adjusted coefficient of determination is $R_{a d j}^{2}=96.8 \%$ while the standard error of calibration Martens and Naes (1989) is $S E C=1.43$ day and the standard error of validation is $S E P=2.16$ days.

Other results obtained from test series with diverse species stored frozen up to 300 days are shown in Table 1. These measurements were made with a commercial TDR (Tektronix TDS 8000). In general the prediction of the storage time using a time domain reflectometer in combination with principle component analysis leads to good results.

Although a graphical presentation is not given, it can be stated, that QIM-scores could also be adequately described by PCA and PCR. QIM-scores between 1 and 25 could be predicted with an SEP of approximately 3 units. However, much of the variance of the QIM-scores can be attributed to the fact that those values are determined by the assesments of human individuals.

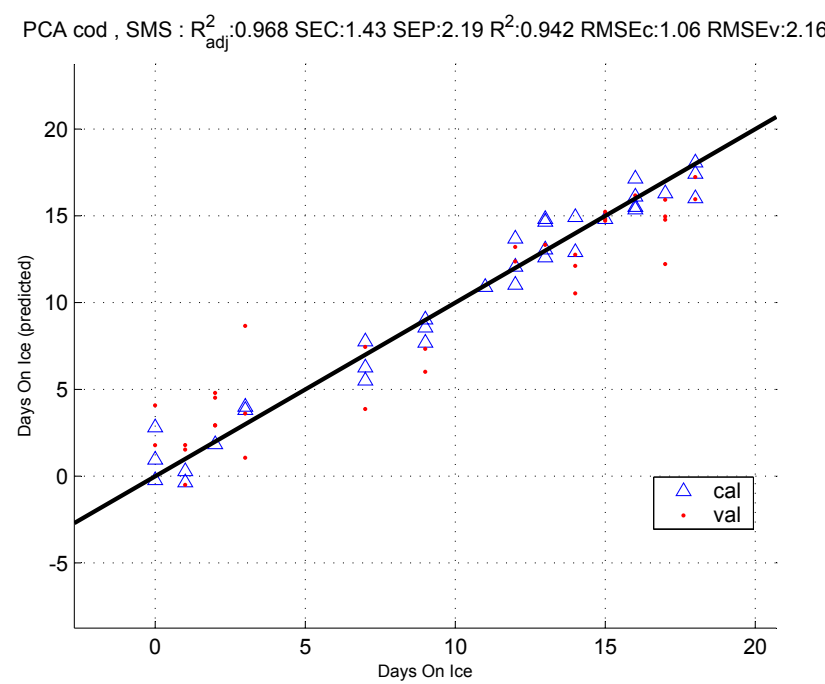

Fig. 5. Prediction of the storage time of cod stored on ice.

\section{Conclusions}

This paper has presented a new approach for the determination of certain properties of natural materials, which were considered as dielectric mixtures. Instead of deriving the required information from measured permittivity values using the physical model of a dielectric mixture formula, a completely different and novel procedure is outlined. A time-step function is applied to the material via an open ended coaxial probe, sampled in a window around the steepest ascent, and the sampling values are then directly processed together with known calibration values by using principal component analysis. In the next step, the calibration is reproduced in a regression equation using some of the principal components. Subsequently unknown measurement values deliver new PCs, which after insertion into the regression equation, are used for the prediction of the unknown properties. The new approach has been demonstrated with the help of a dedicated time domain spectrometer. As examples of the kind of information required the storage time both on ice and in the frozen state, of fish samples was presented. Storage time of cod on ice could be predicted with a standard error of prediction of $\mathrm{SEP}=2.16$ days for a maximum storage period of approximately 18 days. Similar accuracy was obtained for frozen samples of cod which were stored up to 300 days for which the SEP was between typically 30 and 35 days.

It has to be pointed out, that the described approach of extracting information from dielectric measurements is very general and not at all restricted to the described examples. It is also neither restricted to the open ended line nor confined to reflection measurements. Nearly every influence, that expresses in a change of the dielectric relaxation spectrum, can be acquired that way with high accuracy. The physical mechanisms within dielectric mixtures do not have to be known. Also, no empirical or semi-empirical mixture models have to be developed. In fact, the desire to find dielectric mixture 
formulas for natural dielectrics, which are omnipresent in industrial processes, has lead to stagnation in the area of dielectric sensors in the past. It is hoped to overcome this with the widespread application of the new approach.

Acknowledgement. This study has been carried out with financial support from the Commission of the European Communities, Fifth Framework Programme, specific RTD programme Quality of Life and Management of Living Resources, project QLK1-2001-01643, "A New Method For Measurement Of The Quality Of Seafood". It does not necessarily reflect the Commission's views and in no way anticipates its future policy in this area. The other partners in this project are BFAFi,Hamburg, Swedish SIK, Gothenburg, Sweden, CSIC, Madrid, Spain, and IPIMAR, Lisbon, Portugal to whom we are grateful for the acquisition of many of the results reproduced here.

\section{References}

Mulvey, J.: Sampling Oscilloscope Circuits, Oreg., USA, 1970.

Frye, G. J.: Sampling System, Patent US3629731, Portland, Oreg., USA, 1971.

Käs, G. and Pauli, P.: Mikrowellentechnik, Franzis, Munich, Germany, 1991.

Schimmer, O. and Knöchel, R.: A Handheld TDR-System with a fast System Rise-Time and a high Resolution Bandwidth for Moisture Measurements in the Microwave Frequency Range, ISEMA proceedings, 5, 176-177, Rotorua, New Zealand, 2003.

Kent, M. and Anderson, D.: Dielectric studies of added water in poultry meat and scallops, Journal of food engineering, 28, 239259, 1996.

Martens, H. and Naes, T.: Multivariate Calibration, Chichester, UK, John Wiley and Sons, 1989. 